Taxonomía y sistemática

\title{
Análisis morfométrico de dos poblaciones de Anopheles (Anopheles) calderoni (Diptera: Culicidae) del suroccidente colombiano
}

\section{Morphometric analysis of two populations of Anopheles (Anopheles) calderoni (Diptera: Culicidae) from southwestern Colombia}

\author{
Julián Rodríguez-Zabala ${ }^{\mathrm{a}, *}$, Ranulfo González ${ }^{\mathrm{b}}$, Margarita M. Correa ${ }^{\mathrm{a}}$ y Giovan F. Gómez ${ }^{\mathrm{a}}$ \\ ${ }^{a}$ Grupo de Microbiología Molecular, Escuela de Microbiología, Universidad de Antioquia, Calle 70 N. ${ }^{\circ}$ 52-21, Medellín, Colombia \\ ${ }^{\mathrm{b}}$ Facultad de Ciencias Naturales y Exactas, Universidad del Valle, Calle 13 \# 100-00, Ed 320, Cali, Colombia
}

Recibido el 5 de octubre de 2015; aceptado el 4 de marzo de 2016

Disponible en Internet el 4 de agosto de 2016

\begin{abstract}
Resumen
Anopheles calderoni, una especie registrada para Colombia y Ecuador en el 2010, es considerada un vector de malaria en Perú y un vector potencial en la costa pacífica colombiana. Dado el reciente registro de $A$. calderoni, poco se conoce acerca de su variabilidad morfológica y genética en el territorio colombiano. Como una primera aproximación, en este estudio se evaluó la variabilidad morfométrica alar de $A$. calderoni de 2 localidades del occidente colombiano, Guadalajara de Buga y San Andrés de Tumaco. Se midieron 8 variables morfométricas del ala de 76 hembras (Buga $\mathrm{n}=36$, Tumaco $\mathrm{n}=40$ ) y se compararon mediante análisis univariado y multivariado. La comparación pareada de las medidas de los caracteres del ala entre las 2 poblaciones reveló diferencias estadísticamente significativas $(p<0.05)$. El análisis discriminante, con base en estos caracteres, asignó con una baja precisión $(<60 \%)$ los especímenes a su población geográfica original. Las diferencias en el tamaño alar entre las poblaciones de A. calderoni pueden deberse a características microambientales de cada localidad más que a diferencias genéticas. El conocimiento de la estructura poblacional de A. calderoni es relevante para el diseño de estrategias de control vectorial dirigidas.

Derechos Reservados @ 2016 Universidad Nacional Autónoma de México, Instituto de Biología. Este es un artículo de acceso abierto distribuido bajo los términos de la Licencia Creative Commons CC BY-NC-ND 4.0.
\end{abstract}

Palabras clave: Anopheles; Morfometría tradicional; Variación morfológica; Malaria

\begin{abstract}
Anopheles calderoni, a species recorded in Colombia and Ecuador in 2010, is considered a malaria vector in Peru and a potential vector in the Colombian Pacific Coast. Given the recent report of A. calderoni, little is known on its morphological and genetic variability in the Colombian territory. As a first approach, this study evaluated wing morphometric variation on A. calderoni of 2 localities from western Colombia, Guadalajara de Buga and San Andrés de Tumaco. Eight morphometric characters of the wing were measured for 76 females $($ Buga $n=36$, Tumaco $n=40)$ that were compared using univariate and multivariate analyzes. Pairwise comparison of wing character measures between the 2 populations showed statistically significant differences $(p<.05)$. Discriminant analysis based on these characters, assigned with low accuracy $(<60 \%)$ each specimen to its original geographical population. Wing size differences between these A. calderoni populations may be due to micro-environmental characteristics of each locality rather than to genetic differences. The knowledge on A. calderoni population structure is relevant for the design of targeted vector control strategies.

All Rights Reserved (C 2016 Universidad Nacional Autónoma de México, Instituto de Biología. This is an open access item distributed under the Creative Commons CC License BY-NC-ND 4.0.
\end{abstract}

Keywords: Anopheles; Traditional morphometrics; Morphological variation; Malaria

\footnotetext{
* Autor para correspondencia.

Correo electrónico: perseus027@ gmail.com (J. Rodríguez-Zabala).

La revisión por pares es responsabilidad de la Universidad Nacional Autónoma de México.
} 


\section{Introducción}

Anopheles calderoni (Diptera: Culicidae), una especie del subgénero Anopheles, presenta caracteres morfológicos muy similares a los de otras 3 especies de la serie Arribalzagia, como Anopheles punctimacula, A. malefactor y A. guarao (González et al., 2010). De estas, A. guarao aún no se ha registrado en Colombia (Montoya et al., 2011). La serie se encuentra en continua revisión debido a los problemas taxonómicos y al desconocimiento de aspectos importantes de su bionomía y distribución geográfica (Loaiza et al., 2013). En Perú, A. calderoni es reconocido como un vector de importancia local en el occidente peruano, donde se confunde con A. punctimacula y es el segundo en abundancia intradomicilio (Calderón, Fernández y Valle, 1995). En Venezuela se reportó A. calderoni coexistiendo con A. punctimacula (Rubio-Palis y Moreno, 2003). Por su parte, en el territorio colombiano se registró a A. calderoni por primera vez en el 2010 en el Valle del Cauca (González et al., 2010). Con base en este nuevo registro, se realizó una revisión de especímenes de museo provenientes de varias localidades de Colombia, identificados originalmente como A. punctimacula y A. malefactor, evidenciando que algunos de ellos correspondían a A. calderoni. Posteriormente, A. calderoni fue detectado en el departamento de Nariño con infección natural por Plasmodium falciparum, pero su importancia epidemiológica aún debe ser confirmada (Naranjo-Díaz, Altamiranda, Luckhart, Conn y Correa, 2014).

La morfometría tradicional es una herramienta de fácil aplicación por personal entrenado, bajo costo y presenta un gran potencial para la exploración de la diversidad de especies (Cazorla, 2009); ha sido de gran utilidad para esclarecer problemas taxonómicos en especies de difícil diagnóstico, permitiendo diferenciar poblaciones de insectos de importancia médica como Triatoma dimidiata (Bustamante et al., 2004); describir variaciones morfológicas en poblaciones de Phlebotomus papatasi recolectadas a diferentes altitudes (Belen, Alten y Aytekin, 2004) y diferenciar poblaciones de mosquitos Chagasia fajardi provenientes de diferentes ecorregiones (Silva et al., 2012). En mosquitos del género Anopheles, la morfometría tradicional permitió evaluar la variación morfológica de A. pseudopunctipennis en Argentina y Bolivia (Dantur, Liria, Navarro, Rodríguez y Fritz, 2011) y distinguir entre especies del subgénero Nyssorhynchus de Colombia que presentan problemas en su identificación por caracteres morfológicos (Calle, Quiñones, Eraso y Jaramillo, 2002).

En Colombia, A. calderoni solo se ha reportado en el occidente del país (González y Carrejo, 2009). Esta región es una de las más biodiversas del mundo; lo atraviesan 2 hotspots de biodiversidad (Myers, Mittermeier, Mittermeier, da Fonseca y Kent, 2000). Comprende al menos 10 ecorregiones con características ecológicas muy heterogéneas (Fund, 2012), entre las cuales esta la ecorregión denominada de Bosques Montanos del Valle del Cauca (BMVC), y la ecorregión los Bosques Húmedos del Chocó/Darién (BHCD) (Dinerstein et al., 1995), en las cuales se encuentran las localidades del presente estudio. Diferencias ambientales entre ecorregiones podrían conducir a la diferenciación fenotípica de poblaciones de Anopheles (Motoki, Suesdek, Bergo y Sallum, 2012) y tener un impacto en su capacidad vectorial (Ameneshewa y Service, 1996), aspecto relevante para la implementación de estrategias de control vectorial para esta especie. En este estudio se evaluó la variabilidad morfológica alar de especímenes de A. calderoni de 2 localidades, Guadalajara de Buga y San Andrés de Tumaco, situadas en las ecorregiones BMVC y BHCD del occidente colombiano, utilizando morfometría tradicional. Se hipotetizaron diferencias morfológicas a nivel del ala entre estas poblaciones que se encuentran en condiciones ambientales contrastantes.

\section{Materiales y métodos}

Las ecorregión BMVC presenta elevaciones entre los 1,000 y $\operatorname{los} 3,000 \mathrm{~m}$ snm. Su vegetación es diversa y varía a lo largo del gradiente altitudinal; su precipitación varía entre 500 y $1,000 \mathrm{~mm}$ por año en la región de la cordillera occidental, a más de 3,000 mm por año en la región de la cordillera central de los Andes (Fund, 2012). La ecorregión BHCD se encuentra entre la costa pacífica y la cordillera occidental de los Andes colombianos, con una elevación entre 1 y 1,000 m snm; su vegetación varía según la latitud y gradiente altitudinal; constituye una de las regiones más lluviosas del mundo, con precipitaciones entre los 4,000 a los 9,000 mm por año (Fund, 2012).

Los mosquitos Anopheles se recolectaron durante los meses de octubre de 2009, enero y abril de 2010, en la localidad de Píndales $\left(01^{\circ} 37^{\prime} \mathrm{N}, 78^{\circ} 44^{\prime} \mathrm{O}\right)$, municipio de San Andrés de Tumaco, Departamento de Nariño (BHCD), y durante mayo de 2009 en la localidad de Laguna del Sonso $\left(3^{\circ} 52^{\prime} \mathrm{N}\right.$, $6^{\circ} 20^{\prime} \mathrm{O}$ ), municipio de Guadalajara de Buga, Departamento de Valle del Cauca (BMVC) (fig. 1). Estas localidades están separadas por la cordillera occidental, a una distancia aproximada de $360 \mathrm{~km}$. San Andrés de Tumaco, una zona endémica para malaria, está ubicado en la costa pacífica a una elevación de $2 \mathrm{~m} \mathrm{snm}$, presenta una temperatura anual promedio de $28^{\circ} \mathrm{C} \mathrm{y}$ una humedad relativa del 76\%. Guadalajara de Buga no es un área endémica para la malaria, se encuentra en la alta montaña a $969 \mathrm{~m} \mathrm{snm}$, presenta temperatura anual promedio de $26^{\circ} \mathrm{C}$ y una humedad relativa del $88 \%$.

Los especímenes se recolectaron usando cebo humano, bajo consentimiento informado, y mediante un protocolo aprobado por el comité de Bioética (Sede de Investigación UniversitariaSIU, Universidad de Antioquia). Además, se realizó una búsqueda activa de mosquitos en reposo en el intradomicilio y en la vegetación del peridomicilio. Los mosquitos se recolectaron por $6 \mathrm{~h}$, entre las 18:00 y 24:00 horas, durante 6 días consecutivos en cada una de las localidades.

La identificación de los especímenes se realizó siguiendo la clave de González y Carrejo (2009) y las descripciones morfológicas de especies de la serie Arribalzagia (Wilkerson, 1990, 1991). Se realizó el montaje del ala derecha e izquierda y de una de las patas posteriores de cada ejemplar, como soporte a la identificación morfológica y para los análisis morfométricos posteriores; el material restante se almacenó en etanol al $95 \%$ para los análisis moleculares. 


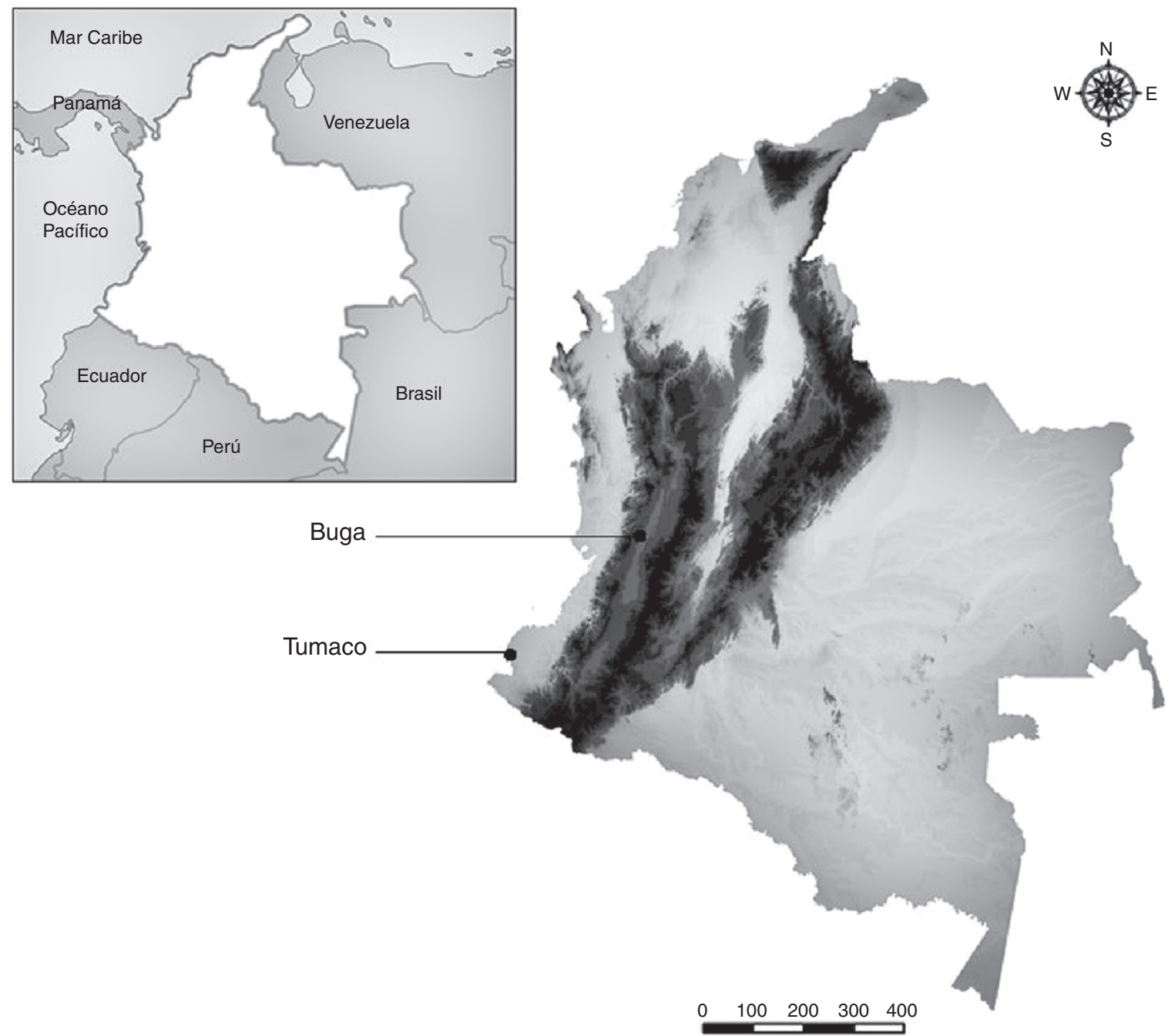

Figura 1. Sitios de recolección de A. calderoni del occidente colombiano. Guadalajara de Buga: Laguna de Sonso (Valle del Cauca). Tumaco: Pindales (Nariño).

\section{Confirmación molecular}

Para confirmar molecularmente el estatus taxonómico de los especímenes identificados como A. calderoni, se seleccionaron aleatoriamente un 5\% de los mismos usando el software EPIDAT 4.0 (OPS/OMS, 2014). Se extrajo el ADN a partir de los abdómenes de los organismos usando un método de precipitación salina (Rosero, Gutiérrez, Cienfuegos, Jaramillo y Correa, 2010). La amplificación de un fragmento del gen citocromo oxidasa subunidad 1 (COI) se realizó utilizando los cebadores universales LCO y HCO (Folmer, Hoeh, Lutz y Vrijenhoek, 1994) y las condiciones previamente estandarizadas (Gómez, Jaramillo y Correa, 2013). Los productos de PCR se enviaron a secuenciar en ambas direcciones y las secuencias se encuentran publicadas en la base de datos del GenBank con los códigos de acceso KF698801-KF698832. La edición y alineamiento de las secuencias se realizó usando el software Geneious R6 (Drummond et al., 2011). Se evaluó la similitud de las secuencias consenso con las reportadas en GenBank mediante un BLAST (http://blast.ncbi.nlm.nih.gov/Blast.cgi), y en la base de datos del Consorcio Código de Barras (http://www.boldsystems.org). Adicionalmente, se compararon las distancias genéticas usando el modelo Kimura 2-parámetros (K2P) con las secuencias COI entre A. calderoni con A. punctimacula y A. calderoni con A. malefactor de Colombia analizados en Gómez, Bickersmith, González, Conn y Correa (2015).

\section{Análisis morfométrico}

Se seleccionaron aleatoriamente 76 especímenes (Buga $\mathrm{n}=36$, Tumaco $\mathrm{n}=40$ ) teniendo en cuenta que sus alas estuvieran en buen estado para los análisis. Se tomaron fotografías del ala derecha de cada ejemplar usando la cámara Moticam 2500 acoplada a un estereomicroscopio triocular OLYMPUS SZ61. Para cada fotografía se digitalizaron 12 puntos de referencia (landmarks), ubicados en puntos de inicio y finalización de manchas o venas (fig. 2), usando el paquete CLIC disponible en: http://mome-clic.com/. Los puntos elegidos se digitalizaron por duplicado por un mismo investigador (J. Rodríguez), para evaluar su repetibilidad. Las mediciones lineales se calcularon usando la opción EDMA del software PAST (tabla 1). 


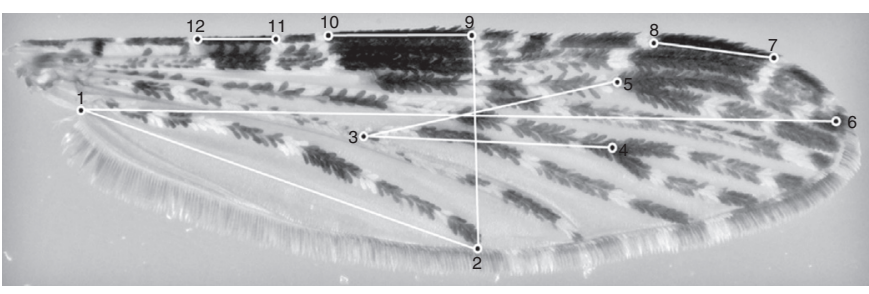

Figura 2. Puntos de referencia seleccionados en el ala de $A$. calderoni. Las líneas trazadas entre puntos de referencia representan las distancias medidas.

Tabla 1

Caracteres morfológicos evaluados en el ala de A. calderoni.

\begin{tabular}{ll}
\hline Distancias & Caracteres medidos \\
\hline $1-2$ & $\begin{array}{l}\text { Distancia desde el lóbulo anal hasta la finalización de la } \\
\text { vena A }\end{array}$ \\
Largo del ala desde el inicio de la vena A hasta \\
finalización de la vena $\mathrm{R}_{2}$ \\
Ancho del ala desde la finalización de la vena A hasta el \\
extremo distal de la mancha SP \\
Distancia entre la intersección vena Cu y mcu y la \\
intersección de las venas $\mathrm{M}_{1}$ y $\mathrm{M}_{2}$
\end{tabular}

A: vena anal; $\mathrm{Cu}$ : vena cubital; mcu: vena mediocubital; $\mathrm{M}_{1}$ : vena media $1, \mathrm{M}_{2}$ : vena media 2; PD: mancha preapical oscura; PSD: presector oscuro; $\mathrm{R}_{2}$ : vena radial $2 ; \mathrm{R}_{3}$ : vena radial 3; $\mathrm{SD}$ : sector oscuro.

La nomenclatura para definir los puntos de referencia para las mediciones lineales corresponde a la descrita en González y Carrejo (2009).

Se calcularon los promedios y desviaciones estándar para todas las variables. Para comparar las mediciones entre las 2 poblaciones, se empleó la prueba no paramétrica de Mann-Whitney, con una confianza del 95\%. Para el análisis multivariado, se aplicó un análisis libre del efecto isométrico sobre las 8 variables, con el cual se corrige el efecto del tamaño sobre las mediciones, siguiendo el método de Burnaby (1966). Se aplicó un análisis de componentes principales para reducir la dimensionalidad de los datos y usar los componentes que aportaron mayor variación. Estos componentes se seleccionaron para la aplicación de un análisis discriminante validado y así evaluar el nivel de diferenciación entre las poblaciones. Todos los análisis estadísticos y representaciones gráficas se realizaron en los programas GraphPad Prism 5.0 y PAST (Hammer, Harper y Ryan, 2008).

\section{Resultados}

El análisis molecular para la confirmación de especie demostró una alta similitud de sus secuencias COI (95\%) al compararlas con las reportados en las plataformas GenBank y BOLD, confirmando así el estatus taxonómico de los especímenes identificados como $A$. calderoni. La distancia genética K2P interespecífica usando el marcador COI entre A. calderoni-A. punctimacula s.s. fue del $9.7 \%$, mientras que entre A. calderoni y A. malefactor fue del $10 \%$.
Tabla 2

Media, desviación estándar e intervalos de confianza del 95\% inferior (ICi) y superior (ICs) de las mediciones expresadas en milímetros.

\begin{tabular}{|c|c|c|c|c|c|c|c|c|}
\hline \multirow[t]{2}{*}{ Distancias } & \multicolumn{4}{|c|}{ Tumaco $(n=40)$} & \multicolumn{4}{|c|}{ Buga $(n=36)$} \\
\hline & Media & DS & $\mathrm{ICi}$ & ICs & Media & DS & $\mathrm{ICi}$ & ICs \\
\hline $1-2$ & 1.93 & 0.11 & 1.89 & 1.96 & 2.17 & 0.09 & 2.14 & 2.20 \\
\hline $1-6$ & 3.67 & 0.20 & 3.60 & 3.73 & 4.18 & 0.15 & 4.12 & 4.23 \\
\hline $2-9$ & 0.97 & 0.06 & 0.95 & 0.99 & 1.10 & 0.05 & 1.08 & 1.12 \\
\hline $3-4$ & 1.15 & 0.07 & 1.13 & 1.17 & 1.42 & 0.07 & 1.29 & 1.33 \\
\hline $3-5$ & 1.25 & 0.08 & 1.22 & 1.27 & 1.31 & 0.06 & 1.39 & 1.44 \\
\hline $7-8$ & 0.54 & 0.06 & 0.52 & 0.55 & 0.60 & 0.06 & 0.58 & 0.61 \\
\hline $9-10$ & 0.59 & 0.05 & 0.57 & 0.61 & 0.70 & 0.05 & 0.67 & 0.71 \\
\hline $11-12$ & 0.32 & 0.05 & 0.30 & 0.33 & 0.37 & 0.05 & 0.34 & 0.38 \\
\hline
\end{tabular}

Todas las variables analizadas mostraron diferencias estadísticamente significativas. U Mann-Whitney $(p<0.05)$.

Tabla 3

Clasificación Jacknife con base en características alares de 2 poblaciones de A. calderoni.

\begin{tabular}{lll}
\hline Localidad & $\mathrm{n}$ clasificados $/ \mathrm{n}$ total & \% clasificación \\
\hline Tumaco & $24 / 40$ & 60 \\
Buga & $23 / 36$ & 63
\end{tabular}

n clasificados: número de individuos correctamente clasificados por población. n total: número total de individuos por población. \% clasificación: porcentaje de especímenes correctamente clasificados con respecto al total por población.

El análisis descriptivo mostró un tamaño promedio mayor para todas las mediciones en los especímenes de Buga (alta montaña), en comparación con los de Tumaco (costa pacífica) (tabla 2). La comparación pareada de las mediciones reveló diferencias estadísticamente significativas para todas las variables analizadas en las 2 poblaciones $(p<0.05)$; dichas diferencias son resultado del efecto isométrico. El análisis de componentes principales mostró que los 3 primeros componentes aportaban el $94 \%$ de la variación, lo que sugirió un efecto importante del tamaño global. El análisis discriminante con validación cruzada (Jacknife), una vez corregido el efecto isométrico del tamaño alar, clasificó correctamente solo el $60 \%$ de los individuos de Tumaco (costa pacífica) y el 63\% de los de Buga (alta montaña), a sus respectivas poblaciones geográficas (tabla 3). Esto indica que no existen diferencias entre los caracteres morfométricos evaluados, excepto por las diferencias en el tamaño general del ala. Alas de mayor tamaño resultaron en distancias más grandes en los caracteres evaluados.

\section{Discusión}

El análisis de las mediciones del ala de A. calderoni permitió evidenciar variación en el tamaño, siendo más grandes las alas de los especímenes de Guadalajara de Buga (alta montaña), en relación con los especímenes de Tumaco (costa pacífica), variación que mostró diferencias estadísticamente significativas para todas las mediciones. Distintas hipótesis podrían explicar las diferencias en el tamaño alar. En primer lugar, los insectos de alta montaña podrían desarrollar alas más grandes para sostener el vuelo con eficiencia en condiciones donde el aire es menos denso (Hepburn, Youthed, Illgner, Radloff y Brown, 1998). Si 
tenemos en cuenta que la localidad de Buga se encuentra ubicada a una altitud de $969 \mathrm{~m}$ snm y Tumaco a $2 \mathrm{~m}$ snm, esto podría explicar las diferencias en el tamaño entre las 2 poblaciones de $A$. calderoni analizadas. El efecto de la altitud sobre el tamaño de las alas se ha encontrado en diferentes insectos (Dillon, Frazier y Dudley, 2006), incluidos mosquitos del género Culex, donde los especímenes que se encuentran en hábitats ubicados a mayores altitudes muestran una relación positiva con respecto al tamaño de sus alas (Demirci, Lee, Lanzaro y Alten, 2011). Esto se ha interpretado como una respuesta adaptativa, donde desarrollar alas más grandes supondría un menor gasto energético para mantener el vuelo en hábitats con una mayor elevación (Dillon et al., 2006). Adicionalmente, las variaciones en el tamaño alar halladas entre las 2 poblaciones de $A$. calderoni podrían estar reflejando una variación clinal de acuerdo a la regla de Bergmann, la cual indica que los ejemplares que habitan a mayores altitudes tienden a tener un mayor tamaño que los provenientes de altitudes más bajas (Marcondes et al., 1999). Sin embargo, para apoyar esta hipótesis en $A$. calderoni se requerirá de la evaluación de especímenes recolectados a través de un gradiente altitudinal. Otra posible explicación podría ser el efecto que tienen las condiciones del hábitat en que se desarrollan las larvas de Anopheles, lo cual influye directamente en el tamaño del mosquito adulto (Araujo, Gil y E-Silva, 2012). Diversos factores microambientales afectan el crecimiento de los mosquitos, como lo son la disponibilidad de alimento, la densidad poblacional del criadero y los diferentes periodos de sequía y lluvia (Jirakanjanakit et al., 2007). Dado que en este estudio se trabajó con poblaciones de campo, dichos efectos microambientales no pueden ser descartados.

La relación entre tamaño corporal y capacidad vectorial es compleja y hay evidencias contrastantes. Por ejemplo, investigaciones en condiciones de laboratorio muestran que las hembras más grandes de $A$. darlingi presentan una mayor supervivencia y tasa de picadura en comparación con las hembras más pequeñas, características que favorecen la infección del mosquito con parásitos Plasmodium spp. y su posterior transmisión al hospedero humano (Araujo et al., 2012). Estudios realizados con poblaciones de A. gambiae han evidenciado resultados similares (Aboagye-Antwi y Tripet, 2010; Manoukis et al., 2006). En contraste, otros estudios en A. gambiae han descrito que la proporción de hembras infectadas con esporozoitos era mayor en mosquitos de tamaño intermedio en comparación con los mosquitos de tamaño más grande o pequeños (Lyimo y Koella, 1992). Anopheles calderoni es un vector de importancia local en el occidente peruano, y en Colombia se detectó infectado naturalmente por $P$. falciparum en el municipio de Tumaco (Naranjo-Díaz et al., 2014). Estudios de laboratorio controlados, bajo un diseño experimental que permita evaluar especímenes de A. calderoni de las localidades de Buga y Tumaco con cepas de Plasmodium spp., podrían proporcionar una respuesta más precisa a la relación entre el tamaño corporal de $A$. calderoni y su capacidad vectorial.

La baja diferenciación de los caracteres morfométricos evaluados en A. calderoni podría ser el reflejo de flujo génico entre estas 2 poblaciones que no permite la fijación de diferencias fenotípicas a nivel del ala (Fairley, Renaud y Conn, 2000;
Gómez, Márquez, Gutiérrez, Conn y Correa, 2014), o mecanismos de canalización fuertes sobre estos caracteres que limitan su variación morfológica (Liefting, Hoffmann y Ellers, 2009). Estudios preliminares de análisis mitocondrial de A. calderoni de Colombia apoyan la primera hipótesis (Gómez et al., 2015).

En conclusión, se encontró variabilidad fenotípica en el tamaño alar entre las poblaciones de A. calderoni como resultado de posibles diferencias microambientales. La evaluación del efecto de cada variable ambiental, como altitud, temperatura, dieta y densidad larval, sobre la variabilidad de los caracteres métricos evaluados bajo condiciones controladas de laboratorio en isofamilias de $A$. calderoni de ambas localidades permitiría estimar el componente genético y ambiental de cada carácter. Dado el bajo número de poblaciones de $A$. calderoni analizadas en este trabajo, se sugiere incrementar su número para conocer a mayor escala la variabilidad fenotípica alar de esta especie y así optimizar la muestra de poblaciones a evaluar en los estudios genéticos, para incluir aquellas que presenten mayores diferencias. Lo anterior permitiría avanzar en el conocimiento de la estructura genética de poblaciones de esta especie, información útil para el diseño de estrategias de control vectorial.

\section{Agradecimientos}

Estudio anidado a un proyecto financiado por el Comité para el Desarrollo de la Investigación-CODI, Universidad de Antioquia, Código N. ${ }^{\circ}$ 8700-689 y recibió apoyo para su ejecución del proyecto CODI CIMB 049-2012. GFG recibió financiamiento del Departamento Administrativo de Ciencia, Tecnología e Innovación-COLCIENCIAS, Colombia.

\section{Referencias}

Aboagye-Antwi, F. y Tripet, F. (2010). Effects of larval growth condition and water availability on desiccation resistance and its physiological basis in adult Anopheles gambiae sensu stricto. Malaria Journal, 9, 225.

Ameneshewa, B. y Service, M. W. (1996). The relationship between female body size and survival rate of the malaria vector Anopheles arabiensis in Ethiopia. Medical and Veterinary Entomology, 10, 170-172.

Araujo, M., Gil, L. H. y Silva, A. (2012). Larval food quantity affects development time, survival and adult biological traits that influence the vectorial capacity of Anopheles darlingi under laboratory conditions. Malaria Journal, 11, 261.

Belen, A., Alten, B. y Aytekin, A. M. (2004). Altitudinal variation in morphometric and molecular characteristics of Phlebotomus papatasi populations. Medical and Veterinary Entomology, 18, 343-350.

Burnaby, T. P. (1966). Growth-invariant discriminant functions and generalized distances. Biometrics, 22, 96-110.

Bustamante, D. M., Monroy, C., Menes, M., Rodas, A., Salazar-Schettino, P. M., Rojas, G., et al. (2004). Metric variation among geographic populations of the Chagas vector Triatoma dimidiata (Hemiptera: Reduviidae: Triatominae) and related species. Journal of Medical Entomology, 41, 296-301.

Calderón, G., Fernández, R. y Valle, J. (1995). Especies de la fauna anofelina, su distribución y algunas consideraciones sobre su abundancia e infectividad en el perú. Revista Peruana de Epidemiología, 8, 5-23.

Calle, D. A., Quiñones, L. M., Eraso, H. F. y Jaramillo, N. (2002). Morphometric discrimination of females of five species of Anopheles of the subgenus Nyssorhynchus from southern and northwest Colombia. Memórias do Instituto Oswaldo Cruz, 97, 1191-1195. 
Cazorla, D. (2009). Multivariate morphometric differentiation between females of two cryptic species of Lutzomyia subgenus Helcocyrtomyia (Diptera: Psychodidae). Revista Colombiana de Entomología, 35, 197-201.

Dantur, J., Liria, J., Navarro, J., Rodríguez, R. y Fritz, G. (2011). Morphometric variability of Anopheles pseudopunctipennis (Diptera: Culicidae) from different ecoregions of Argentina and Bolivia. Florida Entomologist, 94, 428-438.

Demirci, B., Lee, Y., Lanzaro, G. C. y Alten, B. (2011). Altitudinal genetic and morphometric variation among populations of Culex theileri Theobald (Diptera: Culicidae) from northeastern Turkey. Journal of Vector Ecology, 31, 197-209.

Dillon, M. E., Frazier, M. R. y Dudley, R. (2006). Into thin air: physiology and evolution of alpine insects. Integrative and Comparative Biology, 46, 49-61.

Dinerstein, E. D. M., Olson, D. J., Graham, A. L., Webster, S. A., Primm, M. P. y Bookbinder, G. L. (1995). Una evaluación del estado de conservación de las ecorregiones terrestres de América Latina y el Caribe. Washington, D.C.: Banco Mundial y Fondo Mundial para la Naturaleza.

Drummond, A. J., Ashton, B., Buxton, S., Cheung, M., Cooper, A., Duran, C. et al. (2011). Research software for biologists, not computer scientists. Recuperado 15 Sep 2014 de: http://www.geneious.com/

Fairley, T. L., Renaud, T. M. y Conn, J. E. (2000). Effects of local geographic barriers and latitude on population structure in Anopheles punctipennis (Diptera: Culicidae). Journal of Medical Entomology, 37, 754-760.

Folmer, O. B. M., Hoeh, W., Lutz, R. y Vrijenhoek, R. (1994). DNA primers for amplification of mitochondrial cytochrome c oxidase subunit I from diverse metazoan invertebrates. Molecular Marine Biology and Biotechnology, 3, 294-299.

Fund, W. (2012). Ecoregions of Colombia. Recuperado 20 Nov 2013 de: http://www.eoearth.org/view/article/178725

Gómez, G. F., Bickersmith, S. A., González, R., Conn, J. E. y Correa, M. M. (2015). Molecular taxonomy provides new insights into Anopheles species of the Neotropical Arribalzagia. PLoS OnE, 10, e0119488. http://dx.doi.org/10.1371/journal.pone.0119488

Gómez, G. F., Jaramillo, L. y Correa, M. M. (2013). Wing geometric morphometrics and molecular assessment of members in the Albitarsis Complex from Colombia. Molecular Ecology Resources, 13, 1082-1092.

Gómez, G. F., Márquez, E., Gutiérrez, L., Conn, J. E. y Correa, M. M. (2014). Geometric morphometric analysis of Colombian Anopheles albimanus (Diptera: Culicidae) reveals significant effect of environmental factors on wing traits and presence of a metapopulation. Acta Tropica, 135, 75-85.

González, R. y Carrejo, N. (2009). Introducción al estudio taxonómico de Anopheles de Colombia: claves y notas de distribución. Cali: Programa Editorial Universidad del Valle.

González, R., Carrejo, N., Wilkerson, R. C., Alarcon, J., Alarcon-Ormasa, J., Ruiz, F., et al. (2010). Confirmation of Anopheles (Anopheles) calderoni Wilkerson, 1991 (Diptera: Culicidae) in Colombia and Ecuador through molecular and morphological correlation with topotypic material. Memórias do Instituto Oswaldo Cruz Cruz, 105, 1001-1009.

Hammer, O., Harper, D. A. T. y Ryan, P. D. (2008). PAST: Paleontological Statistics Software Package for education and data analysis. Palaentología Electrónica, 4-9.

Hepburn, H. R., Youthed, C., Illgner, P., Radloff, S. E. y Brown, R. E. (1998). Production of aerodynamic power inmountain honeybees (Apis mellifera). Naturwissenschaften, 85, 389-390.
Jirakanjanakit, N., Leemingsawat, S., Thongrungkiat, S., Apiwathnasorn, C., Singhaniyom, S., Bellec, C., et al. (2007). Influence of larval density or food variation on the geometry of the wing of Aedes (Stegomyia) aegypti. Tropical Medicine \& International Health, 12, 1354-1360.

Liefting, M., Hoffmann, A. y Ellers, J. (2009). Plasticity versus environmental canalization: population differences in thermal responses along a latitudinal gradient in Drosophila serrata. Evolution, 63, 1954-1963.

Loaiza, J. R., Scott, M. E., Bermingham, E., Sanjur, O. I., Rovira, J. R., Dutari, L. C., et al. (2013). Novel genetic diversity within Anopheles punctimacula s.1.: phylogenetic discrepancy between the barcode cytochrome c oxidase I (COI) gene and the rDNA second internal transcribed spacer (ITS2). Acta Tropica, 128, 61-69.

Lyimo, E. O. y Koella, J. C. (1992). Relationship between body size of adult Anopheles gambiae s.1. and infection with the malaria parasite Plasmodium falciparum. Parasitology, 104, 233-237.

Manoukis, N. C., Toure, M. B., Sissoko, I., Doumbia, S., Traore, S. F., DiukWasser, M. A., et al. (2006). Is vector body size the key to reduced malaria transmission in the irrigated region of Niono, Mali? Journal of Medical Entomology, 43, 820-827.

Marcondes, C. B., Lozovei, A. L., Falqueto, A., Brazil, R. P., Galati, E., Aguiar, G., et al. (1999). Influence of altitude, latitude and season of collection (Bergmann's rule) on the dimensions of Lutzomyia intermedia (Lutz \& Neiva, 1912) (Diptera, Psychodidae, Phlebotominae). Memórias do Instituto Oswaldo Cruz, 94, 693-700.

Montoya, J., Solarte, A. Y., Giraldo-Calderon, G. I., Quiñones, L. M., Ruiz, L. F., Wilkerson, R. C., et al. (2011). Malaria vector species in Colombia a review. Memórias do Instituto Oswaldo Cruz, 106, 223-238.

Motoki, M. T., Suesdek, L., Bergo, E. S. y Sallum, M. A. M. (2012). Wing geometry of Anopheles darlingi Root (Diptera: Culicidae) in five major Brazilian ecoregions. Infection, Genetics and Evolution, 12, 1246-1252.

Myers, N., Mittermeier, R., Mittermeier, C., da Fonseca, G. y Kent, J. (2000). Biodiversity hotspots for conservation priorities. Nature, 403, 853-858.

Naranjo-Díaz, N., Altamiranda, M., Luckhart, S., Conn, J. E. y Correa, M. (2014). Malaria vectors in ecologically heterogeneous localities of the Colombian Pacific region. PLoS One, 9. http://dx.doi.org/10.1371/ journal.pone.0103769

OPS/OMS (2014). Epidat: Programa para análisis epidemiolóxico de datos. Versión 4.1. Consellería de Sanidade, Xunta de Galicia, España; Organización Panamericana da saúde (OPS-OMS); Universidade CES, Colombia. Recuperado 10 Sep 2015 de: http://dxsp.sergas.es

Rosero, D., Gutiérrez, L., Cienfuegos, A., Jaramillo, L. y Correa, M. (2010). Optimización de un procedimiento de extracción de ADN para mosquitos anofelinos. Revista Colombiana de Entomología, 36, 260-263.

Rubio-Palis, Y. y Moreno, J. (2003). Primer registro de Anopheles (Anopheles) calderoni (Diptera: Culicidae) en Venezuela. Entomotropica, 18, 159-161.

Silva, J., Carvajal, A. L., Serra-Freire, N., Guimaraes, A., Sarmento, J. y Alencar, J. (2012). Morphometric analysis of Chagasia fajardi (Diptera: Culicidae) populations in Brazil. Revista Colombiana de Entomología, 32, 276-282.

Wilkerson, R. C. (1990). Redescriptions of Anopheles punctimacula and A. malefactor (Diptera: Culicidae). Journal of Medical Entomology, 27, 225-247.

Wilkerson, R. C. (1991). Anopheles (Anopheles) calderoni n.sp., a malaria vector of the Arribalzagia series from Perú (Diptera culicidae). Mosquito Systematics, 23, 25-38. 